\title{
The Oxford SWIFT Spectrograph: first commissioning and on-sky results
}

\author{
Niranjan Thatte ${ }^{* a}$, Matthias Tecza ${ }^{\mathrm{a}}$, Fraser Clarke ${ }^{\mathrm{a}}$, Timothy Goodsall ${ }^{\mathrm{a}}$, Lisa Fogarty ${ }^{\mathrm{a}}$, Ryan \\ Houghton $^{\mathrm{a}}$, Graeme Salter ${ }^{\mathrm{a}}$, Nicholas Scott ${ }^{\mathrm{a}}$, Roger L. Davies ${ }^{\mathrm{a}}$, Antonin Bouchez ${ }^{\mathrm{b}}$, Richard Dekany ${ }^{\mathrm{b}}$ \\ ${ }^{a}$ Dept. of Astrophysics, University of Oxford, Keble Road, Oxford, OX1 3RH, U.K.; \\ ${ }^{\mathrm{b}}$ California Institute of Technology Optical Observatories (COO), 1200 E. California Blvd., \\ Pasadena, CA 91125, U.S.A.
}

\begin{abstract}
The Oxford SWIFT spectrograph, an I \& z band (6500-10500 $\AA$ ) integral field spectrograph, is designed to operate as a facility instrument at the 200 inch Hale Telescope on Palomar Mountain, in conjunction with the Palomar laser guide star adaptive optics system PALAO (and its upgrade to PALM3000). SWIFT provides spectra at $R(\equiv \lambda / \Delta \lambda) \sim 4000$ of a contiguous two-dimensional field, $44 \times 89$ spatial pixels (spaxels) in size, at spatial scales of $0.235^{\prime \prime}, 0.16^{\prime \prime}$, and $0.08^{\prime \prime}$ per spaxel. It employs two $250 \mu \mathrm{m}$ thick, fully depleted, extremely red sensitive $4 \mathrm{k} \times 2 \mathrm{k}$ CCD detector arrays (manufactured by LBNL) that provide excellent quantum efficiency out to $1000 \mathrm{~nm}$.

We describe the commissioning observations and present the measured values of a number of instrument parameters. We also present some first science results that give a taste of the range of science programs where SWIFT can have a substantial impact.
\end{abstract}

Keywords: integral field; spectrograph; fully depleted, thick CCDs; adaptive optics; image slicer; laser guide star.

\section{INTRODUCTION}

The Oxford SWIFT instrument [5], first conceived in 2003, and now in routine operation at the 200 inch Hale Telescope on Palomar Mountain, is an I \& z band integral field spectrograph (IFS) that builds upon the success of the highly popular SINFONI near-infrared IFS at the ESO-VLT. It fills a niche, combining the power of adaptive optics (AO) [6] with a high throughput IFS to give unprecedented sensitivity and resolution at wavelengths up to $1050 \mathrm{~nm}$.

SWIFT and the 200 inch Hale Telescope make an attractive combination, as the latter is one of the few $4 \mathrm{~m}$ class telescopes worldwide that has concrete plans to provide a second generation laser guide star (LGS) AO system that would provide substantial correction of the atmospheric turbulence at the SWIFT wavelengths $(650-1050 \mathrm{~nm})$. The implementation of SWIFT+LGS-AO was always planned to occur in stages. At first installation and commissioning, SWIFT could already benefit significantly from the $\times 2$ improvement in ensquared energy (EE) per $0.235^{\prime \prime}$ spaxel from the existing PALMAO system. The subsequent planned upgrade of PALMAO to PALM 3000 [1] would provide Strehl ratio exceeding $10 \%$ at the SWIFT wavelengths, thus providing improved spatial resolution and sensitivity performance unrivalled by any other AO-IFS combination.

\section{INSTRUMENT PARAMETERS}

In simple terms, SWIFT can be viewed as an I \& z band complement to AO assisted near-infrared integral field spectrographs, such as OSIRIS@Keck, NIFS@Gemini or SINFONI@VLT. It provides 4000 simultaneous spectra, arranged in 2:1 aspect ratio field of view (44 $\times 89$ spaxels), so as to optimally allow "nodding-on-IFU", enabling almost perfect subtraction of the night sky background. Although the sky emission at the SWIFT wavelengths $(650-1050 \mathrm{~nm})$ is substantially lower than in the near-infrared (thus providing a corresponding sensitivity benefit), the benefit of using nodding-on-IFU has been well demonstrated at Palomar. SWIFT has a fixed spectral format, dispersion is achieved by

* thatte@astro.ox.ac.uk; Tel: +44 1865 273412; Fax: +44 1865 273390; http://astroweb1.physics.ox.ac.uk

Ground-based and Airborne Instrumentation for Astronomy III, edited by lan S. McLean,

Suzanne K. Ramsay, Hideki Takami, Proc. of SPIE Vol. 7735, 77357Y · @ 2010

SPIE · CCC code: $0277-786 X / 10 / \$ 18 \cdot$ doi: $10.1117 / 12.857484$

Proc. of SPIE Vol. $773577357 Y-1$ 
high performance volume phase holographic gratings (VPHGs) in the twin spectrographs, each using a thick $(250 \mu \mathrm{m})$, fully-depleted, $4 \mathrm{k} \times 2 \mathrm{k}$ LBNL CCD detector [2], with unprecedented red sensitivity and extremely low fringing, especially valuable longward of $900 \mathrm{~nm}$. The two detectors are read out synchronously, and are labelled master and slave for the two spectrograph channels, based on the controller configuration. SWIFT observations can use one of three spaxel (spatial pixel) scales, selectable "on-the-fly", depending on the spatial resolution provided by the AO system. These spaxel scales are $0.235^{\prime \prime}, 0.16 "$ and 0.08 ", corresponding to fields of view of $10.3 " \times 20.9 ", 7 " \times 14.2 "$, and $3.5 " \times$ 7.1" respectively. In one dimension (along the long axis of the field of view) it would be possible to bin on CCD, providing enhanced sensitivity. For 2 to 1 binning, the corresponding spaxels on sky would be rectangular, $0.47 " \times$ 0.235 " for the largest scale. The spectral resolution is $\approx 4000$ across the spectral range, covering the entire wavelength range in a single exposure.

\section{UNIQUENESS}

First and foremost, SWIFT is an IFS, providing all the benefits of integral field spectroscopy over traditional long slits, or Fabry-Perot type instruments. These are (but not limited to):

- "point-and-shoot" capability does not require exact information of object position (esp. useful for emission line objects) or prior knowledge of source structure and extent.

- Delivering a "homogenous" data cube, with uniform atmospheric and PSF conditions over the whole data cube, allowing accurate line ratio or line $\mathrm{v} / \mathrm{s}$ continuum diagnostics

- no need to compromise between wide slits (slit effect, low spectral resolution) and narrow slits (atmospheric dispersion, accurate positioning, slit loss)

- efficient use of telescope time, in contrast to scanning techniques.

- "correct" 2D kinematic and dynamic information, for detailed scientific analysis.

Image slicer: SWIFT provides an IFS capability without compromising on throughput. This is largely due to a novel demagnifying image slicer (designed by M. Tecza (Univ. of Oxford) [4] and D. Freeman (Kidger Optics Associates) that converts the two-dimensional field of view into a pair of long slits with only two flat mirrors and one (AR coated) doublet in the light path (see Figure 1). This builds upon the heritage of SPIFFI (the SINFONI IFS) [6] which was the highest throughput near-IR spectrograph amongst the VLT instrumentation suite, and shatters the myth that integral field spectroscopy has a substantial throughput cost associated with the added capability.

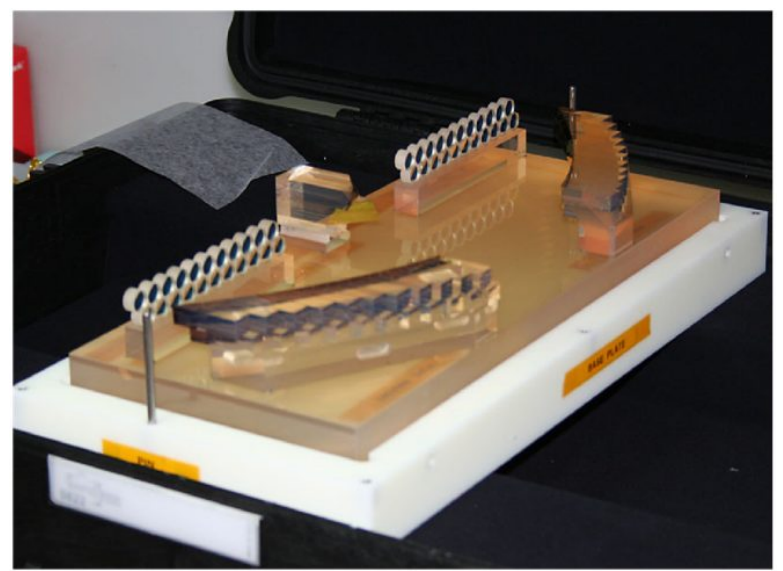

Figure 1: Photograph showing the SWIFT slicer, fully assembled as a monolithic unit on a Zerodur base plate. The slicer light path involves only two reflections and a single AR coated transmissive doublet.

Throughput: The entire SWIFT instrument is designed to achieve a high throughput in a compact opto-mechanical assembly, so that the overall instrument efficiency (from photons above the atmosphere to detected electrons) is $\sim 23 \%$ over most of the band-pass. The high throughput is the second factor contributing to the uniqueness of the instrument.

Novel CCDs: At the red end, the high instrument throughput owes much to a pair of thick $(250 \mu \mathrm{m})$, fully depleted, extremely red-sensitive $4 \mathrm{k} \times 2 \mathrm{k}$ CCD detectors, developed and supplied by LBNL [2]. These devices provide very high QE almost out to the silicon band edge, whilst simultaneously benefiting from low fringing ( $\sim \%$ at $1000 \mathrm{~nm}$ measured 
at the $0.235^{\prime \prime}$ spaxel scale). Compared to most CCD arrays deployed in spectrographs at major observatories around the world (see Figure 2), the LBNL detectors employed in SWIFT represent a sensitivity gain of at least a factor of two, increasing to almost a factor of three at $1000 \mathrm{~nm}$. The unprecedented red sensitivity of the instrument clearly outlines a scientific niche - studies of red-shifted emission lines that lie in the wavelength range $850-1030 \mathrm{~nm}$.

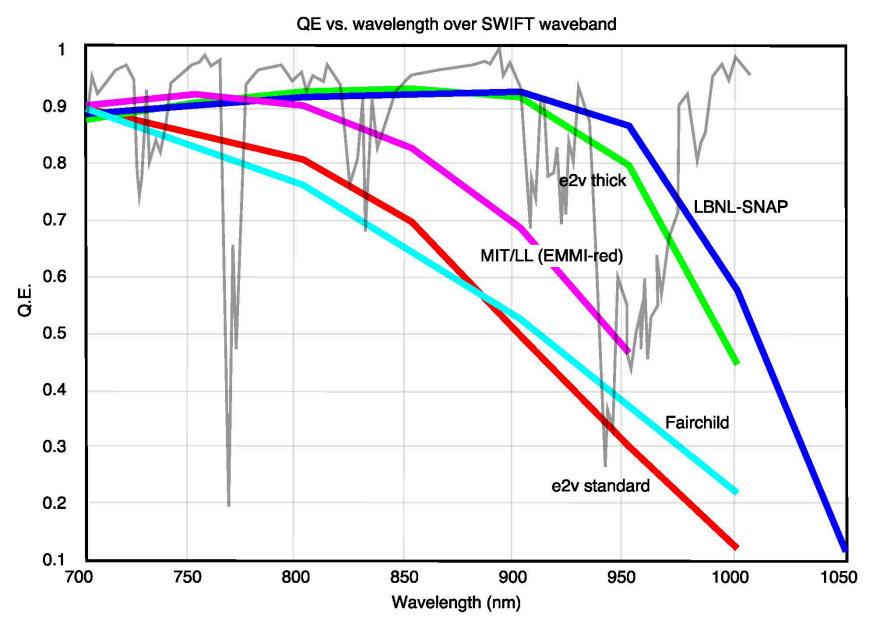

Figure 2: QE curves for various thick and thin CCD detectors installed in spectrographs at major observatories worldwide.

The thin gray line represents the atmospheric transmission.

AO and EE gains: Although several integral field spectrographs operational at major observatories around the world cover the SWIFT wavelength range, very few, if any, are assisted by LGS-AO. Most AO systems are capable of providing very good performance at near-IR wavelengths (Strehl $50-70 \%$ at $\mathrm{K}$ band), dwindling to Strehl ratios of a few percent in the $J$ band. Although these systems typically do not provide a measurable Strehl improvement at wavelengths shortward of $1 \mu \mathrm{m}$, they still do provide a significant gain in ensquared energy (EE), and thus, in sensitivity, that can be exploited to advantage.

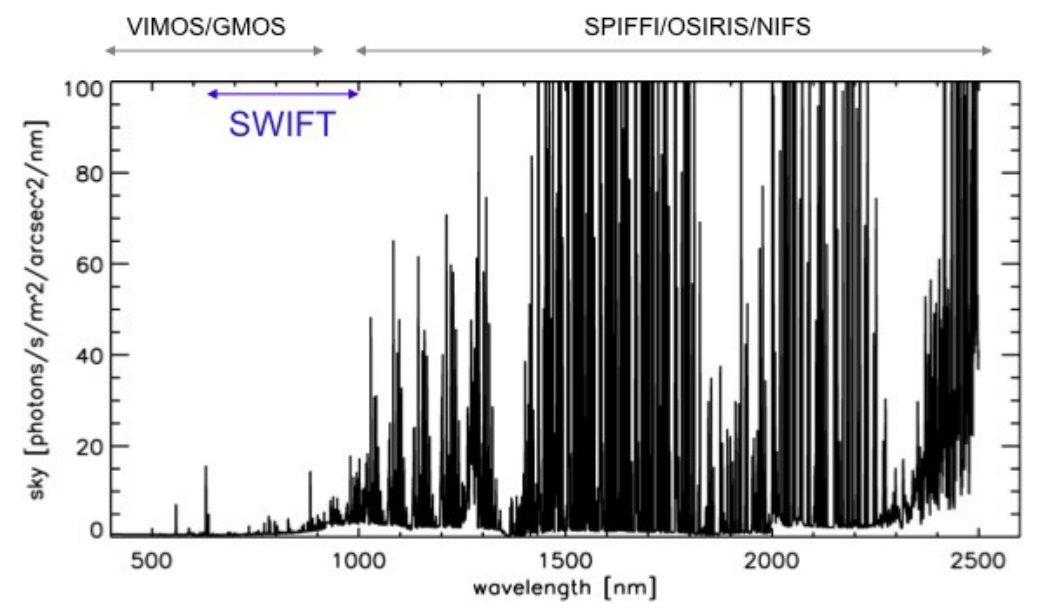

Figure 3: Night sky emission line background as a function of wavelength. The dramatic difference between the near-IR and the SWIFT wavelength range is apparent.

Measured performance of SWIFT with the PALMAO LGS system [3] (see section 5.3 below) shows an EE gain of over a factor of 2 per spaxel in good seeing conditions. This corresponds to a factor of four in exposure time required per target, making a substantial difference in the sample size of typical observing programs. As the AO performance improves, culminating in a second generation system with high order correction and a powerful laser beacon to sample the turbulence with adequately many sub-apertures, SWIFT, with its three choices of spaxel scale, is already set up to fully exploit the capabilities as they are provided. When the AO system is capable of providing appreciable Strehl 
$(>10 \%)$ at the red end of the SWIFT range [2], SWIFT observations will truly be unparalleled, combining large collecting area with high sensitivity and fine spatial resolution.

SWIFT is inherently a spectrograph, and as such, sensitivity in terms of light collected per spaxel should be the primary measure, rather than spatial resolution. It should be kept in mind that even the smallest spaxel scale of SWIFT, at 0.08" per spaxel, is almost a factor of 2 larger than the Hale telescope diffraction limit at the red end of the SWIFT bandpass, and a factor of 4 away from the spaxel size required for Nyquist sampling. The goal is to gain in EE and sensitivity, rather than achieving the ultimate spatial resolution possible.

Sky background: In comparison with most near-IR integral field spectrographs, which do benefit substantially from AO assisted observations, SWIFT has the benefit of much fainter sky background. Figure 3 shows the difference in the night sky emission strength over the SWIFT wavelength range, in comparison to the J \& $\mathrm{H}$ bands that are covered by other instruments such as OSIRIS, SINFONI and NIFS. The corresponding sensitivity gain is obvious, although the night sky background at the SWIFT wavelengths is bright enough to warrant need for careful sky background subtraction.

\section{COMMISSIONING}

\subsection{Commissioning run and observations}

The SWIFT shipping crates arrived at Palomar Mountain on Sep $17^{\text {th }}, 2008$. After a couple of weeks of intensive work by the team, unpacking, cooling down, testing and aligning, SWIFT was installed on the PALMAO bench at the Cassegrain focus of the 200 inch Hale telescope on Oct $11^{\text {th }}$, 2008. Four commissioning nights followed, unfortunately the weather was particularly bad, and the telescope dome could be opened on only one of the four nights, just enough to allow observations of some standard stars, and a couple of bright commissioning targets. Figure 4 shows some of the SWIFT team members next to the instrument just after successful installation at the telescope.

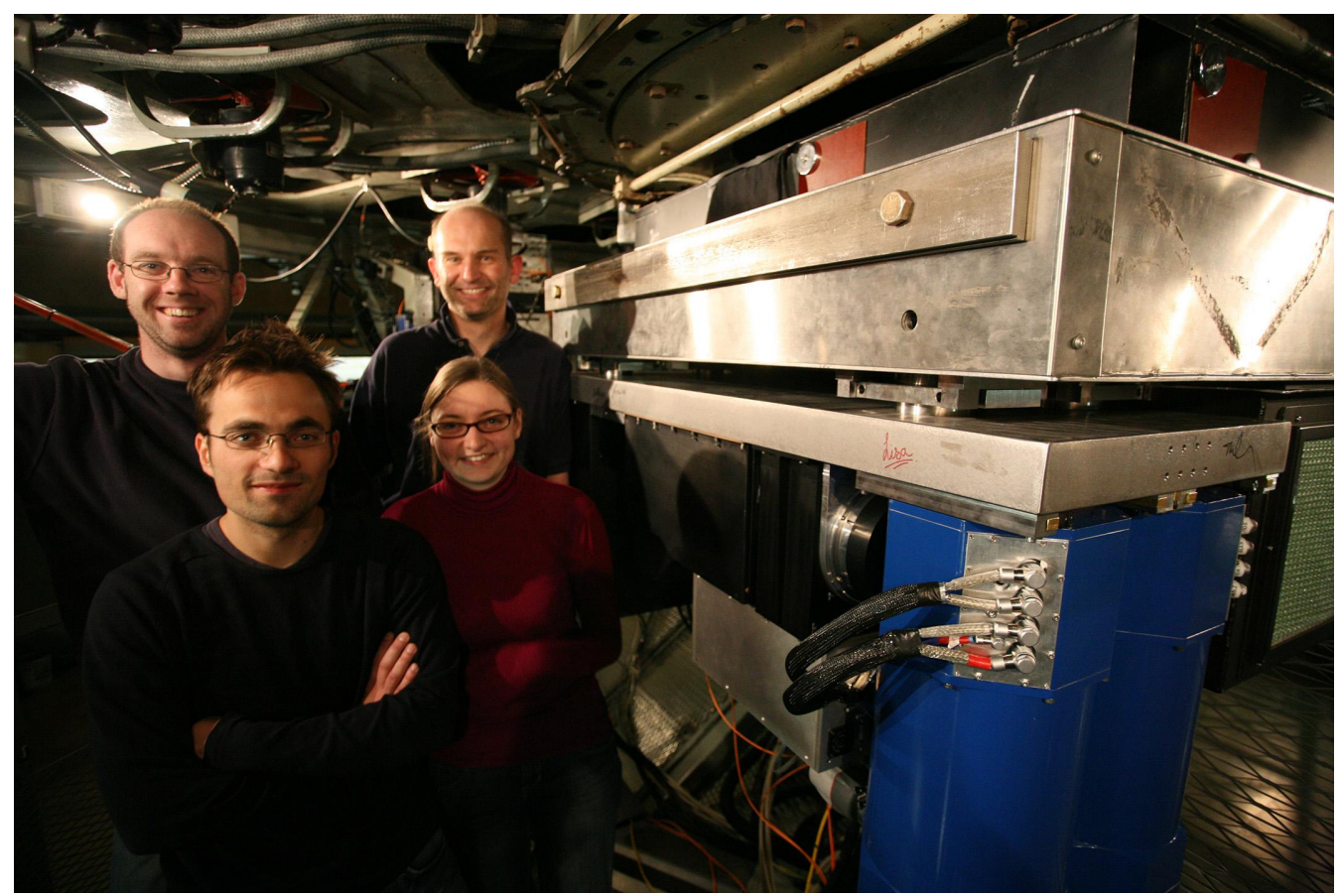

Figure 4: SWIFT team members (clockwise from front-right) L. Fogarty, T. Goodsall, F. Clarke and M. Tecza, next to the instrument mounted to the $\mathrm{AO}$ bench at the Cassegrain focus of the 200 inch Hale Telescope. The blue cryostats house the LBNL CCD detectors.

Due to poor weather during commissioning in October 2008, further commissioning was carried out during part of the science observation nights scheduled in Dec 2008 and Jan 2009, moving the science observations to a best-effort basis. This included some LGS mode commissioning as well. Results are reported in subsequent sections. 


\subsection{Pupil alignment and focus}

First commissioning at the telescope involved, among other things, alignment of the telescope/AO pupil with the instrument pupil, and determining the best focus position for the instrument via reconstructed images. The AO WFS cameras were then set to the same focus setting as the instrument to ensure optimal image quality. Figure 5 shows a through focus sequence - the telescope pupil (with its large central obscuration) is clearly seen in the out-of-focus images.

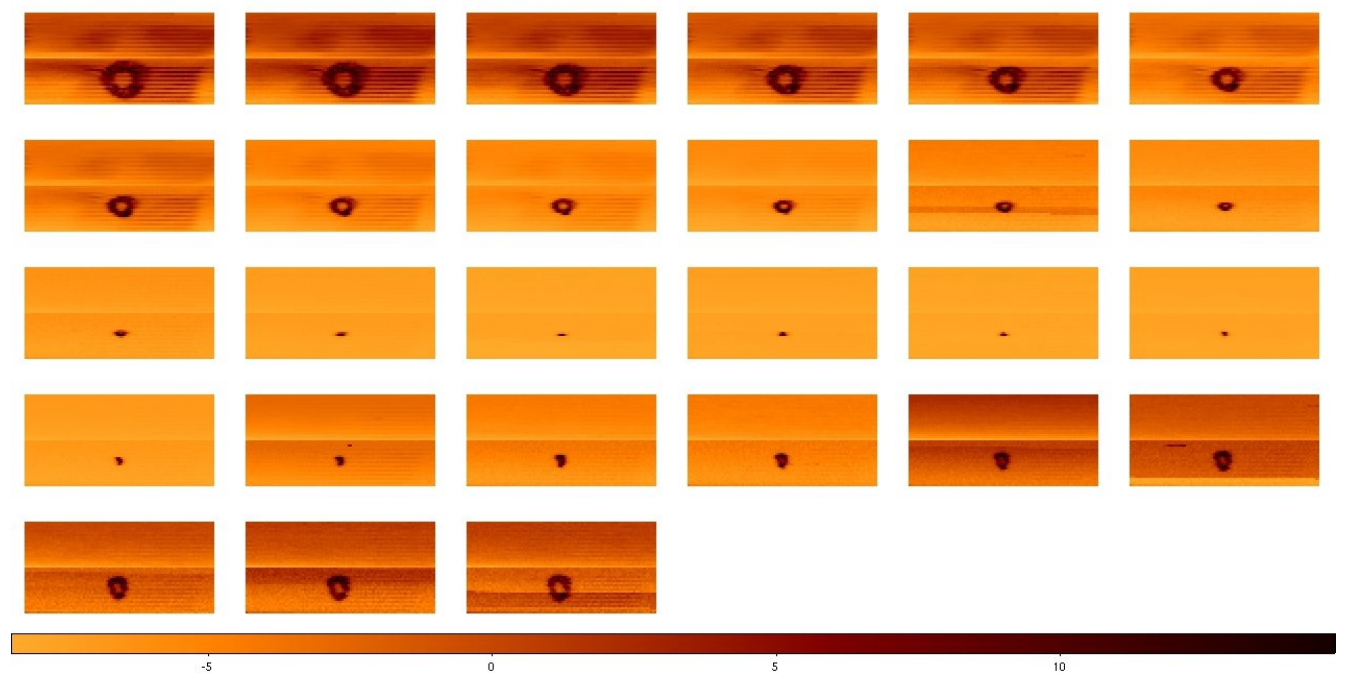

Figure 5: Through focus scans of a point source used to determine the best focus for the instrument at first commissioning.

The displayed plots show the quick look reconstructed images (collapsed data cubes) for a sequence of focus exposures (inside focus at top left, outside focus at bottom right).

\subsection{Throughput and sensitivity}

The total throughput of the SWIFT spectrograph (including atmosphere, telescope, PALMAO, science dichroic, instrument and detector) was measured in May 2009 via observations of Wolf 1346, a spectro-photometric standard star. The plot below (Figure 6) shows the measured throughput of the instrument, including the detector. The assumed throughputs for the telescope and the $\mathrm{AO}$ system are 0.75 and 0.8 respectively, with the latter including a 0.9 dichroic transmission. The black curve is for the master chip (science grade detector), the blue curve is for the slave chip (engineering grade detector). Note that the sensitivity stays high at very long wavelengths, up to $10000 \AA$. Beyond $9000 \AA$, SWIFT is at least twice (and probably thrice) as sensitive as a typical observatory spectrograph with a standard CCD detector array. Note that the atmospheric transmission profile has not been removed from the throughput plot.

Individual exposure times are limited to be $1800 \mathrm{~s}$ long, as the thick chips are more susceptible to cosmic rays than standard CCDs. Typical exposure times are $900 \mathrm{~s}$ for faint targets. Based on the measured throughput, we can compute the instrument sensitivity for line and continuum emission. We use the following values for the telescope, AO and atmosphere when computing the sensitivity: a telescope optics throughput of 0.75 (two Al reflections at 0.875 each), an AO system science throughput of 0.8 (6 reflections at 0.98 (freshly recoated optics), plus one dichroic beamsplitter at 0.9 ), an $\mathrm{AO}$ ensquared energy gain of 1.6 gain in the central $0.235^{\prime \prime}$ pixel due to sharper core (no gain if extraction aperture is $\approx$ seeing disk size), an atmospheric transmission of unity, and a night sky background (under average lunar illumination conditions) of $19.5 \mathrm{mag} / \mathrm{arcsec}^{2}$ in the I band, $18.3 \mathrm{mag} / \operatorname{arcsec}^{2}$ in the $\mathrm{z}$ band.

For the instrument itself, we used the flat part of the measured throughput curve for the combined instrument and detector efficiency. The detector parameters used are read noise of 3.2/4.1 $\mathrm{e}^{-}$per pixel for the two readouts of the master chip, and 4.3/4.1 $\mathrm{e}^{-}$per pixel for the slave chip, dark current of $8 \mathrm{e}^{-}$per $\mathrm{hr}$ per pixel, mean detector gain (for both amplifiers) 0.97 and $1.04 \mathrm{e}^{-} / \mathrm{ADU}$ for the master and slave chips respectively. We assumed 30 minutes (1800 secs) per exposure (DIT), longer exposures are made up of NDIT sub-units DIT seconds long.

With these assumptions, we obtain the following sensitivity numbers for continuum and line sensitivity, respectively. 
- Expected continuum sensitivity: For a $z=22$ mag (Vega) point source, we obtain an SNR of 1.3 in the continuum (per $1 \AA$ spectral pixel at $\mathrm{R} \sim 4000$ ) in a single 30 min exposure, in 1 " seeing.

- Expected line sensitivity: For a source with a line flux of 1e-17 ergs s $\mathrm{cm}^{-2} \operatorname{arcsec}^{-2}$, with a line width of 150 $\mathrm{km} \mathrm{s}^{-1}$, we obtain an SNR of 0.9 in the line a single 30 min exposure per spatial pixel. This corresponds to an SNR of 3.65 per 1 " extraction aperture in the same time.

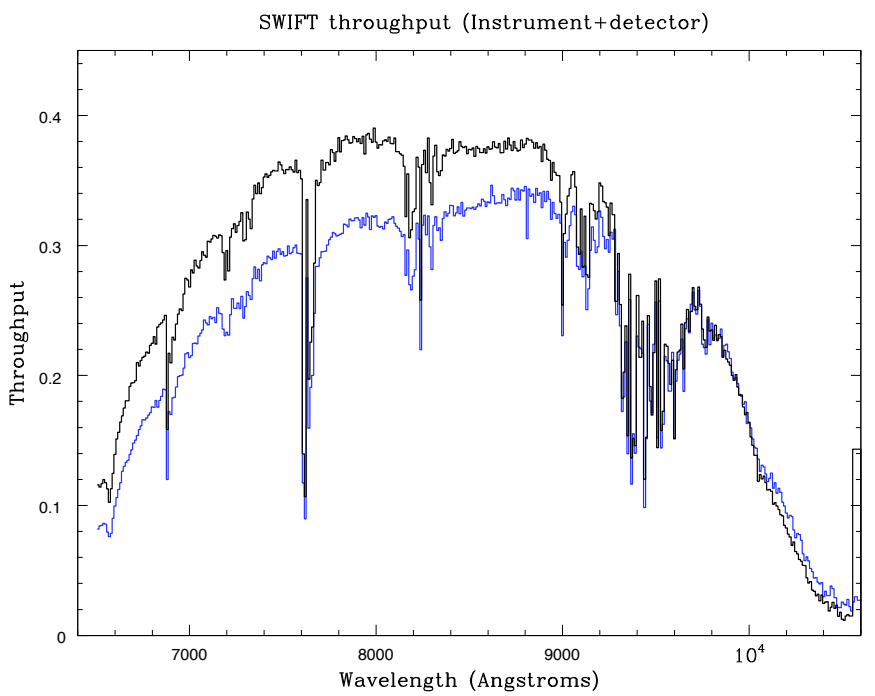

Figure 6: SWIFT measured instrument throughput for master (black line) and slave (blue line) chips

IFS sensitivity computations can be quite complex, as one has to consider both the spatial and spectral properties of the source. For point sources, a substantial gain in sensitivity can be had by combining the flux for all spaxels (spatial pixels), each of which contain the same spectral information. In photon noise dominated situations (either from source or sky background) the gain is typically $\mathrm{N}^{1 / 2}$, where $\mathrm{N}$ is the number of spaxels over which the point source light is spread out.

On the other hand, for very extended sources, the sensitivity can appear to be rather poor, but it should be kept in mind that the quoted numbers are often per spatial and spectral pixel, whereas, typically, one is only interested in information on spatial scales at least as large as the PSF (seeing or AO), and often larger (dictated by source characteristics). In both cases, substantial sensitivity gains are achieved by summing over all spaxels within the extraction aperture (typical gain $\mathrm{N}^{1 / 2}$, where $\mathrm{N}$ is the number of spaxels that are summed over).

\subsection{Operation and Calibration}

SWIFT is operated remotely from computers that are physically located in the data room, and are connected to local electronics in the Cassegrain cage over networks. Communications to instrument control hardware (i.e. motor controller, temperature monitoring, etc) is via a standard 10BaseT Ethernet connection to an RS232 terminal server in the electronics rack. Detector controllers (SDSU) communicate with PCI cards in the host machine via 62.5/125 micron ST fibre links.

The SWIFT instrument control software has been developed in LabVIEW, based around the "ArcVIEW" approach common to current generation Palomar instruments. SWIFT software runs on Linux PCs (Fedora-core). The instrument control software is self-contained, with the only external link being to the PALAO system. This link is implemented by a small C-library handling standard Unix communication commands; socket (), connect (), send(), recv(). TCS commands/responses are sent through the PALAO system. GUI front-ends for all instrument controls are built in LabVIEW.

The current SWIFT detector control software consists of a Python (+GUI) front end to a C library. The detector control software is not yet integrated with the rest of the instrument control software, but we plan to develop an ArcVIEW based module for detector control in the near future. 
User interface: The current SWIFT software provides a quick $(<1 \mathrm{~s})$ image reconstruction tool for target acquisition (RTD). This software displays a 2D image in ds 9 and interacts with the instrument control software allowing the user to "click-and-drag" a target to the desired location on the field of view. Interaction with the PALMAO adaptive optics system (closing and opening the AO loop, small image plane offsets) can also be performed using the SWIFT GUIs. Before the AO system can being used (and even it is not being used for observations), the telescope has to be slewed to a bright nearby star prior to the science observations. The reference star is used to generate a "flatmap" for the AO deformable mirror, which in practice also includes corrections for any static aberrations in the telescope optics (predominantly focus). The flatmap procedure has to be repeated every couple of hours as its terms change with telescope position.

Calibration: Flat field, trace, and wavelength calibration are performed using a dedicated calibration unit located within the instrument, which provides halogen lamp and $\mathrm{Ne} / \mathrm{Ar}$ arc lamp illumination over the full field of view. A third position of the calibration mirror redirects telescope light to an imaging camera, which can be used for acquisition of particularly faint targets. Telluric absorption and flux calibration is performed using spectro-photometric standard stars, the latter requires the star image to be placed on both the master and the slave chips to correctly account for the transmission differences between the two spectrographs.

\subsection{Data reduction pipeline}

A dedicated pipeline is available to reduce SWIFT data, developed by R. Houghton and T. Goodsall. It is based on the spred pipeline developed for the SINFONI instrument at the ESO-VLT, with some important additions such as cosmic ray detection / interpolation. It produces reduced data cubes from which the instrumental signature has been removed, so that the result is bias subtracted, flat fielded, cosmic ray / bad pixel corrected, wavelength calibrated, reformatted into a mosaicked data cube consisting of both master and slave data sets, and illumination corrected.

Recently, a separate pipeline has been developed by R. Houghton, based on the IRAF package, that allows accurate wavelength calibration using night sky emission lines within the science exposure itself, thus removing any effects arising from instrumental flexure. It provides the same functionality as the previous pipeline, but with much improved data quality, resulting from superior calibration and fewer interpolations.

\subsection{LGS commissioning}

The SWIFT LGS AO [3] operation mode was first commissioned during an observing run in Dec 2008. However, weather conditions were uncooperative, and only a limited amount of commissioning could be carried out. We did manage to obtain a data set with the acquisition camera (see Figure 7) that demonstrates the improvement provided by the laser star. There was significant improvement in the PSF width, even at the SWIFT wavelengths, although the atmospheric seeing was less than optimal for adaptive optics observations $(\sim 2 ")$.

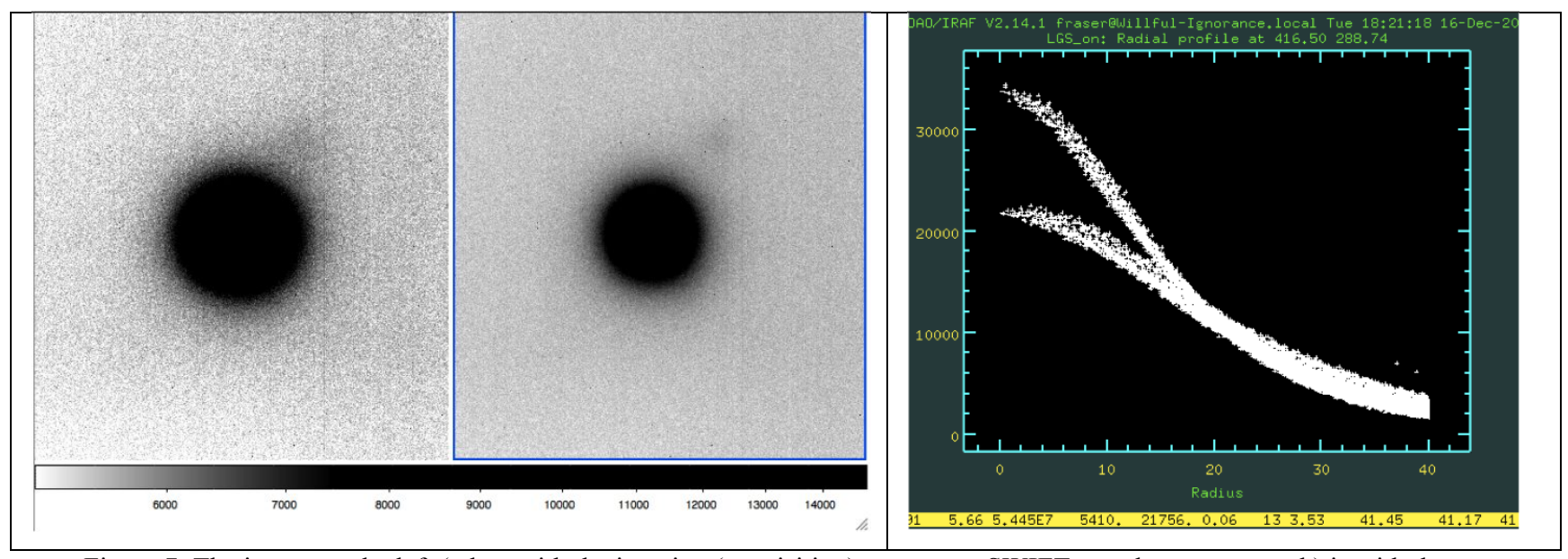

Figure 7: The image on the left (taken with the imaging (acquisition) camera on SWIFT, not the spectrograph) is with the LGS turned off, while the one on the right is with the LGS turned on. Radial profile plots are shown alongside. The substantial improvement in image FWHM is easily seen. 
Due to laser improvement activities, the Palomar LGS was not available in semester 2009A, and we were only able to obtain good quality data on the LGS-AO performance during the observing run in Aug 2009. Following a complete realignment by Ed Kibblewhite in July 2009, the laser was performing very well and producing in excess of $8 \mathrm{~W}$ at the output of the laser (as high as $9.8 \mathrm{~W}$ was measured during the run). The new chirping mode of the laser, which has shown a factor of 1.8 greater photon return, was not used, promising even better performance once it is fully operational. We made no specific tests of LGS return, but quick comparisons with NGS correction showed the laser was equivalent to an R 11 NGS. Most of the science nights on the run enjoyed good natural seeing, with V band FWHM typically around 0.8-1.0". The LGS system worked very well in these conditions, delivering significant gains in FWHM and ensquared energy.

FWHM/Image quality gains: An AO corrected PSF generally consists of two components; a diffraction limited core and a seeing limited halo. The better the high order AO correction, the more light goes into the core and less remains in the halo. Residual tip-tilt errors on top of the high order correction move this core+halo PSF around, smearing out the core in long exposures $(>0.1 \mathrm{~s})$.

Figure 8 shows a comparison of the PSF with LGS off (natural seeing of $\sim 0.75 "$ at $850 \mathrm{~nm}$ ) and LGS on. The core+halo nature of the AO corrected PSF is clear comparing the radial profiles. The core has a FWHM of $\sim 0.4 "$ ", but it is likely to be unresolved at this spaxel scale of $0.16^{\prime \prime}$ (The diffraction-limited core at this wavelength is $\sim 0.04$ "; significantly smaller than the SWIFT spaxel scale of $0.16^{\prime \prime}$ ).

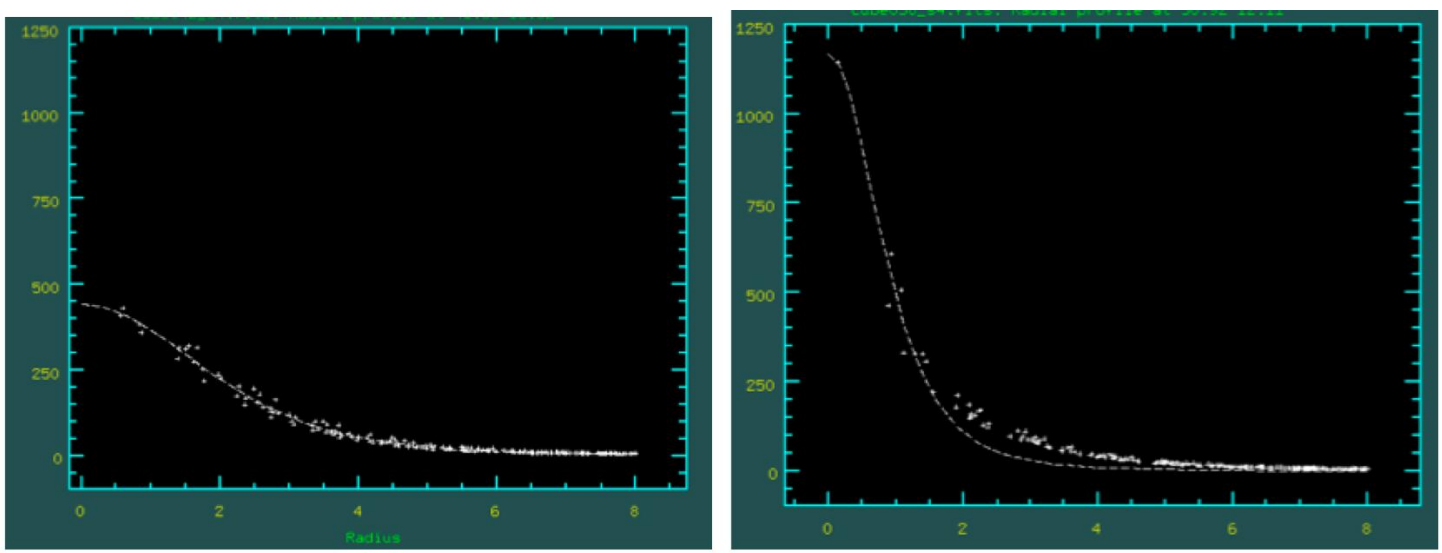

Figure 8: Comparison of LGS off (left) and LGS on (right) radial profiles at 850nm. The sharp core in the LGS image is obvious. Both profiles are plotted on the same scale. 1 spaxel is $0.16^{\prime \prime}$ on the sky

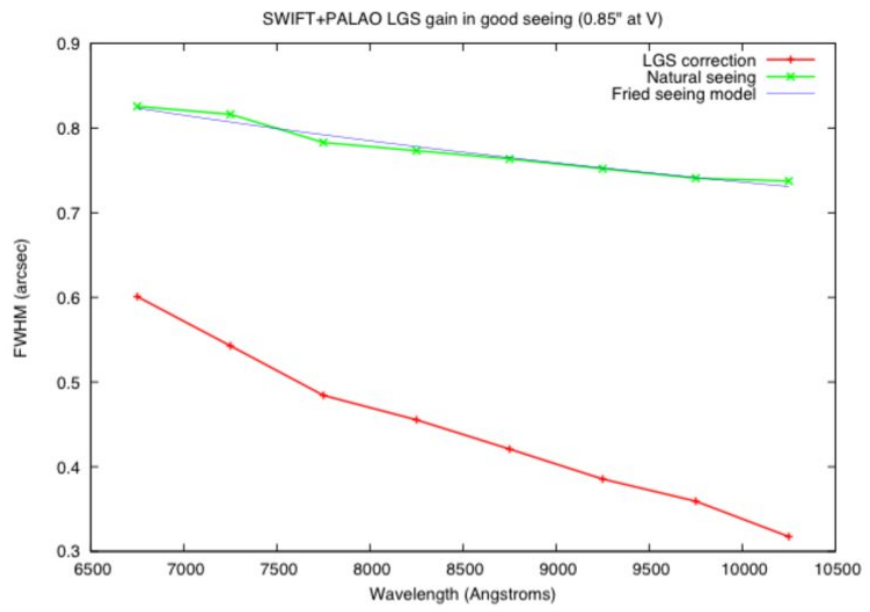

Figure 9: FWHM as a function of wavelength for natural seeing (green upper line) and LGS AO correction (red lower line). The natural seeing behaviour is a good match to a Fried seeing model (FWHM $\propto \lambda^{-1 / 5}$; blue line). 
Figure 9 shows how the LGS corrected FWHM varies as a function of wavelength. As expected, LGS performance is better towards longer wavelengths, but at all points in the SWIFT wavelength range, LGS is reducing the natural seeing FWHM. The natural seeing follows a Fried FWHM $\propto \lambda^{-1 / 5}$ model well.

Ensquared energy gain: For a spectrograph such as SWIFT, ensquared energy is as important, if not more so, than absolute image quality. The ensquared energy (EE) is the amount of flux concentrated in the central $1 \times 1$ or $2 \times 2$ spaxels, relative to the total flux in the PSF. Improvement in EE leads directly to S:N gains for unresolved objects.

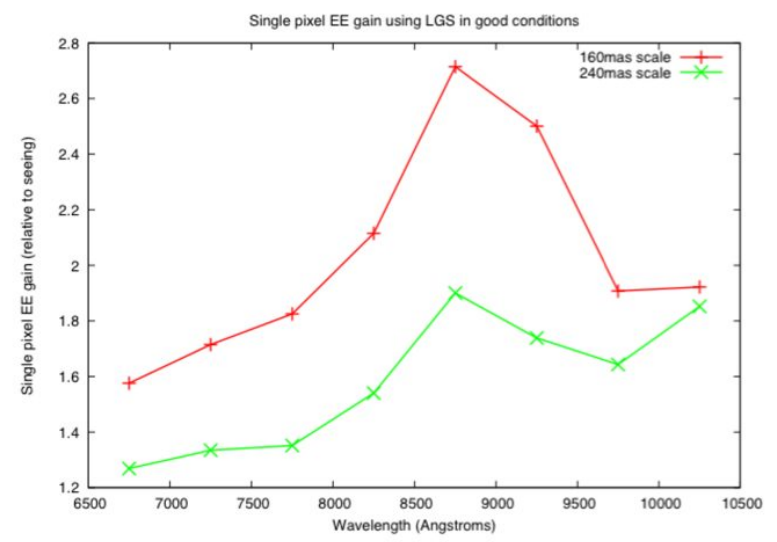

Figure 10: single pixel $(1 \times 1)$ ensquared energy gain with LGS compared to natural $\left(0.85^{\prime \prime}\right)$ seeing. In the 160 mas scale, we gain a factor of 2 in enquared energy over a seeing limited PSF over much of SWIFT's wavelength range

Figure 10 shows the $1 \times 1$ spaxel gain in ensquared energy from LGS observations over natural seeing observations. All of the data were taken in the $0.16^{\prime \prime}$ spaxel scale, and equivalent $0.235^{\prime \prime}$ images/numbers have been resampled from this data. As a result, there is some additional uncertainty on the $0.235^{\prime \prime}$ numbers. LGS gives significant gains over seeing limited observations in terms of EE. In the $0.16^{\prime \prime}$ scale, where we get the biggest benefit from the unresolved LGS core, the central spaxel contains twice as much energy as the seeing limited case over much of SWIFT's wavelength range. The drop off in EE towards the red end of the wavelength range is due to an unresolved image quality problem with SWIFT in the extreme red.

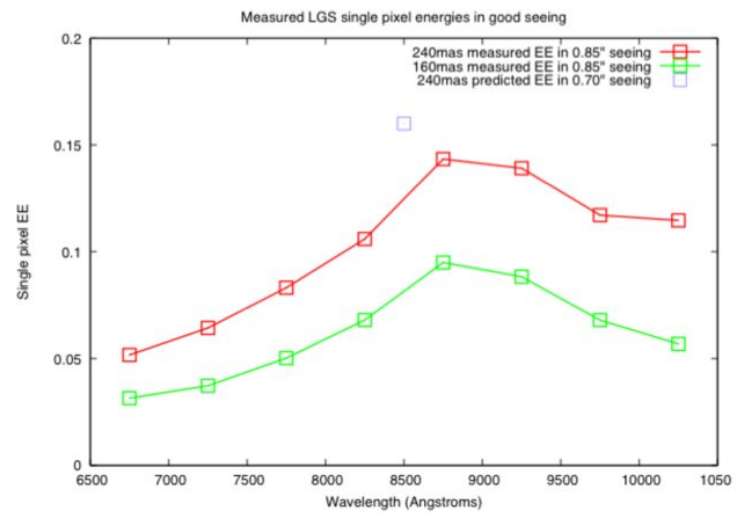

Figure 11: Single spaxel ensquared energies in good conditions ( 0.85 " natural seeing in the V band). The blue point is the predicted EE in excellent conditions $\left(0.7^{\prime \prime}\right.$ at V).

Figure 11 shows the absolute single spaxel ensquared energies for the $0.235^{\prime \prime}$ and $0.16^{\prime \prime}$ spaxel scales over the SWIFT wavelength range. At $850 \mathrm{~nm}, 14 \%$ of the PSF's energy is going into the central spaxel in the $0.235^{\prime \prime}$ scale. This compares with $16 \%$ predicted from simulations in slightly better conditions $(0.7 \mathrm{l} \mathrm{v} / \mathrm{s} 0.85$ " V-band seeing).

Performance in poor conditions: The LGS engineering night at the start of the run had poor seeing (mainly mirror seeing due to a large $\left(10^{\circ} \mathrm{C}\right)$ drop in ambient temperature during the previous day), with FWHM around 3". Somewhat surprisingly the LGS system does deliver significant benefits even in bad conditions. LGS correction reduced the 
FWHM from 3" to 2" (this is almost all due to LGS correction; running with and without a tip-tilt star (V=11) made no difference to the performance).

Efficiency: Open shutter efficiency on science targets was $40 \%$ during the run (excluding weather loss, but including time loss due to SWIFT/AO errors). Efficiency increased each night as we learnt how to use the system (Figure 12) reaching $47 \%$ by the last night (including a 40 minute loss due to an AO crash). The overheads mainly come from LGS acqusition, and $2 \mathrm{amp}$ readout mode employed for the SWIFT CCDs ( $\sim$ readout time of 80 seconds per exposure).

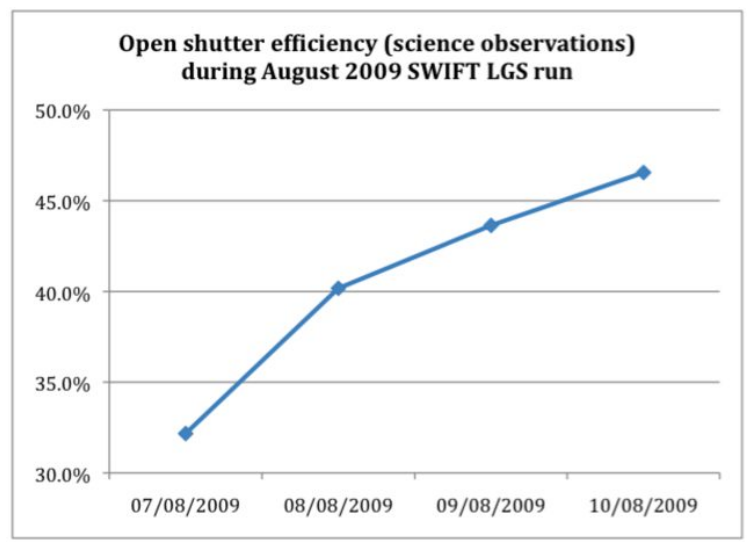

Figure 12: Open shutter efficiency for science observations (i.e. excluding acquisition time) for SWIFT observations during August 2009 LGS run. Efficiency improved through the run as we gained experience with the system

Full moon: One of the target fields was very close to the (full) moon. We did however successfully use the laser as close as 15 degrees from the moon, with the limit being the increased background in the tip-tilt wavefront sensor.

\section{FIRST RESULTS}

In this section we present a brief summary of some of the first observations carried out with the SWIFT spectrograph. The aim is to illustrate the capabilities of the instrument, providing simultaneous spectroscopy of extended objects over a large wavelength range, stretching beyond $1000 \mathrm{~nm}$.

\subsection{Arp 147}

Arp 147 is a spectacular interacting galaxy system comprising of a collisionally-created ring galaxy and an early type companion galaxy, that was recently imaged by the Hubble Space Telescope (see Figure 13). It was observed in January 2009 as part of a bad weather backup SWIFT program to study the kinematics of interacting galaxies. The coarsest scale was used, as seeing was $\sim 2.5^{\prime \prime}$, and no AO correction was possible. Three different pointings were mosaicked together to form the data cube, from which line maps in the emission lines of $\mathrm{H \alpha}$, [NII], [SII] and [SIII], as well as line free continuum images were extracted. Sky subtraction was performed using exposures of blank sky interleaved with the object exposures.

The kinematics observed with SWIFT show a clear signature for an expanding ring, and the expansion can be disentangled from the rotation using the spatially resolved kinematic data. The equivalent width of the H $\alpha$ line conclusively indicates that a young starburst is occurring in the ring, consistent with the HST colours. It is probably triggered by the collision. Other line ratios (Ho/[NII], [SII], and [SII]/[SIII]) allow us to deduce that the eastern half of the ring is more metal rich than the western half, and that the redder region in the south-east corner of the ring is likely to be the nuclear bulge of the disk galaxy that formed the ring. 

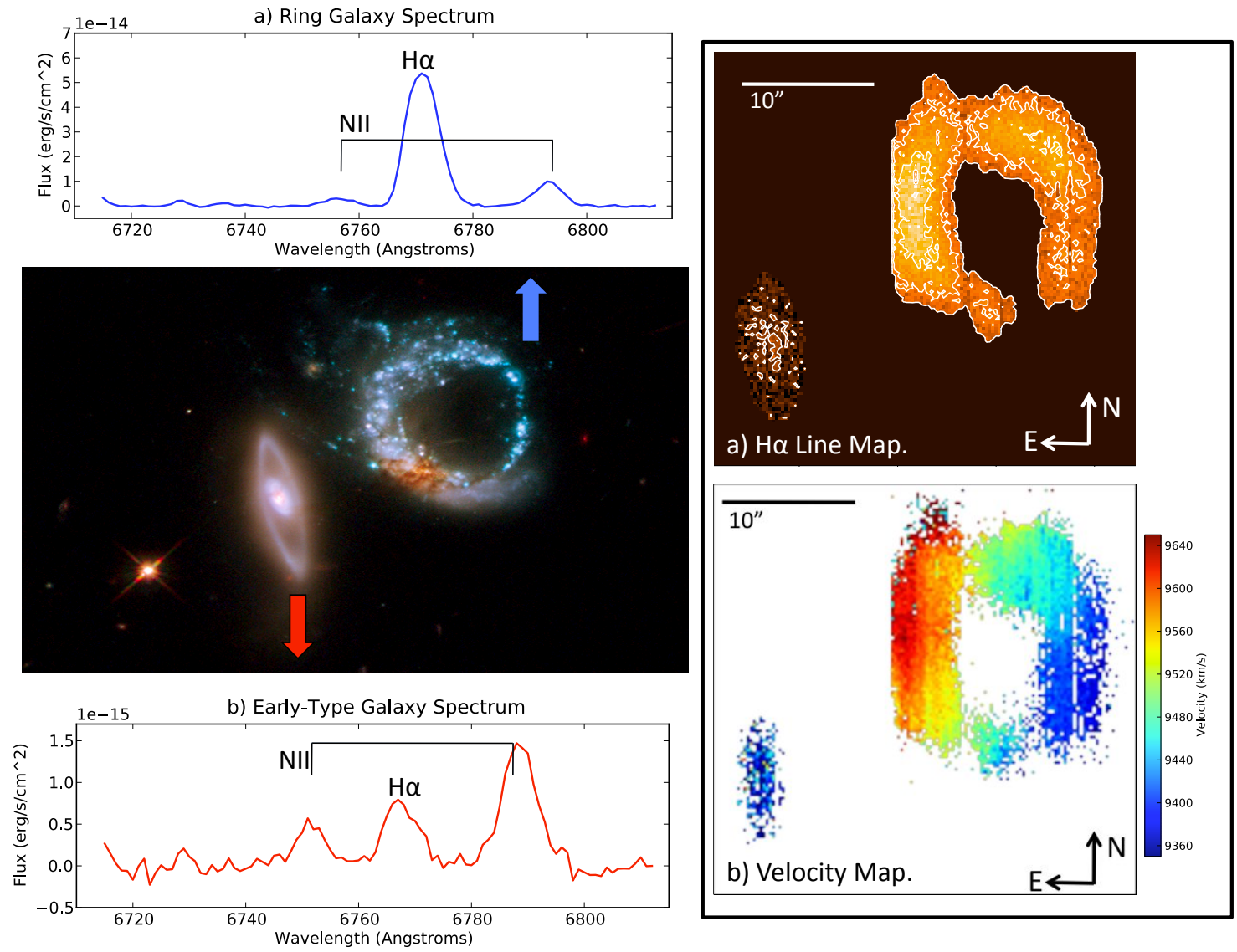

Figure 13: Composite figure showing the wealth of information gained from SWIFT observations of the Arp 147 interacting galaxy system. The HST false-colour image is shown, together with integrated SWIFT spectra of the ring and early type galaxies, in the left hand panels. The right panels show the continuum subtracted H $\alpha$ line map, and the velocity field derived from it.

\subsection{Coma cluster Fundamental Plane}

The program aims to measure the kinematics of early type galaxies in several well-defined luminosity bins spanning the entire luminosity range of early type galaxies in the dense Coma cluster. The SAURON survey indicated that the specific angular momentum of early type galaxies (ETGs) provides a physical classification that relates much more closely to the assembly history of galaxies than the traditional morphological classification of E and S0. By measuring the specific angular momentum of the early type galaxies in a well studied rich cluster we aim to establish their merger history.

Coma is the richest nearby cluster and is the local comparator for studies of the evolution of galaxies in clusters. Coma has extensive HST imaging from which we can derive accurate effective radii and surface brightness. Combining these with SWIFT kinematic measurements ( $\sigma_{e}-$ the velocity dispersion within the effective radius), we can place these galaxies on the Fundamental plane, and derive a dynamical mass-to-light ratio for comparison with that determined from the stellar populations. 


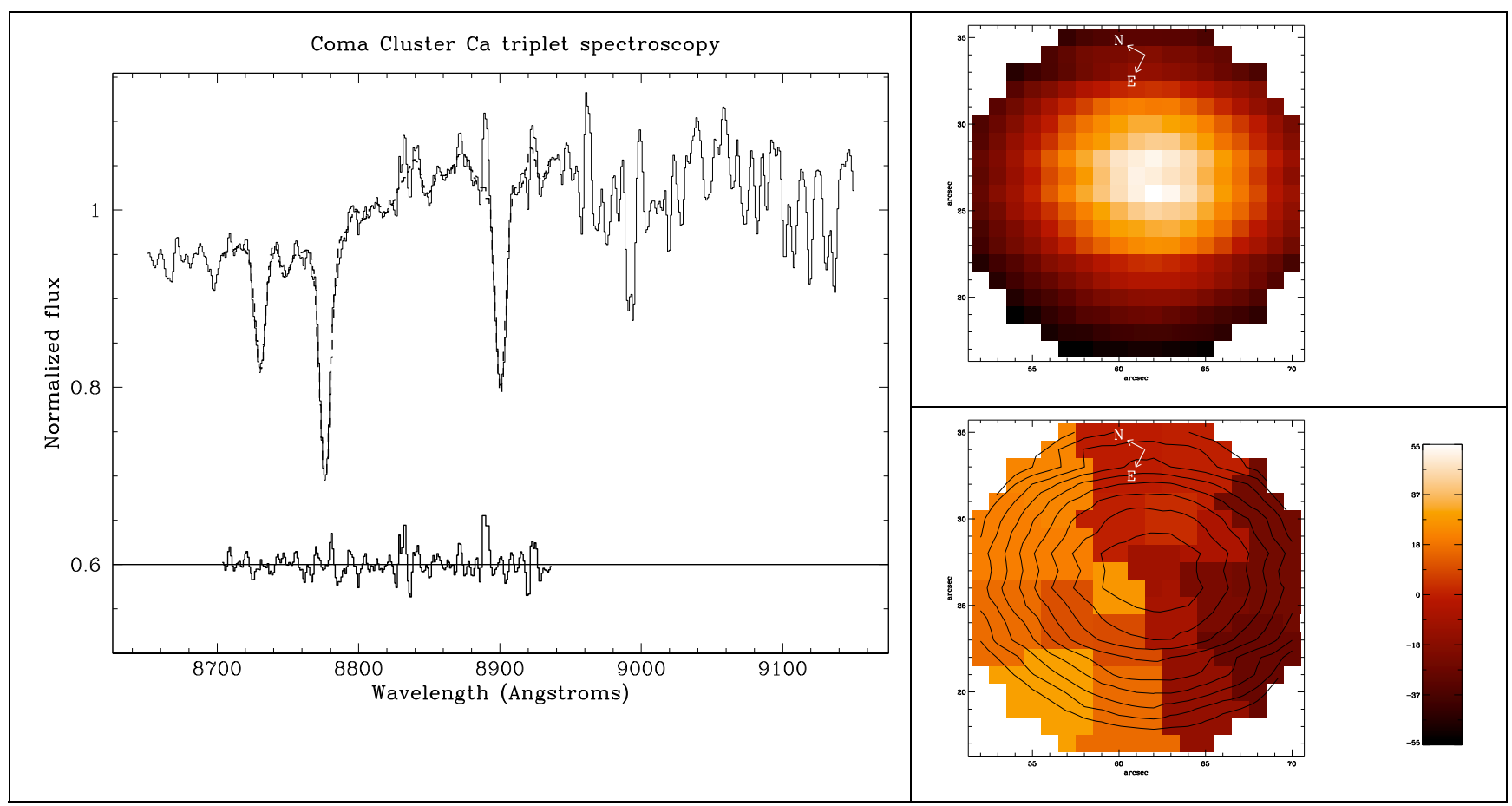

Figure 14: SWIFT spectroscopy of the galaxy PGC044562 in the Coma Cluster. These data are part of a program to establish a more accurate measurement of the fundamental plane for early type galaxies within the Coma cluster. The $\mathrm{Ca}$ triplet absorption feature (left panel) is used to measure the galaxy velocity field (bottom right) and dispersion. The reconstructed continuum image is shown on the top right. Data and analysis courtesy N. Scott and R. Houghton.

\subsection{LGS-AO results}

The SWIFT+LGS run in Aug 2009 had four nights for science observations. The first half of the first night was lost to weather (humidity). The rest of the run was fortunate to have good seeing and clear skies. The nights were split between the SWIFT key project to measure the Tully-Fisher relationship at z 1 (led by S. Kassin and L. Fogarty), and a project to confirm gravitational lenses discovered through the Extensive Galaxy Zoo Lensing Survey (EGZeLenS) program led by A. Verma. Both programmes used the LGS-AO to good effect, with integrations of up to 1200 s being carried out with relative ease (with AO loops continuously closed). None of the targets in either programme would have been possible with NGS-AO.

Figure 15 shows an initial reduction of data from the EGZeLenS project (Verma et al., in prep) The left hand panel shows the SDSS discovery image with the SWIFT field overlaid (the lens candidate is the arc just below the middle of the box). The centre panel shows a collapsed 2-D image of 10 minutes of observations (seeing $0.8^{\prime \prime}-1.0^{\prime \prime}$ ), created from $\sim 100 \mathrm{~nm}$ of the data cube. The arc is faintly detected in this (relatively) broadband image. To show the benefits of the LGS observations, the right panel shows an identical length exposure $(600 \mathrm{~s})$ made without AO (from May 2009, seeing 1.2"$\left.1.4^{\prime \prime}\right)$. The gain in image quality, and commensurate gain in sensitivity with LGS, is clear.

\section{CONCLUSIONS}

The Oxford SWIFT spectrograph is a state-of-the-art I \& $\mathrm{z}$ band integral field spectrograph that is in routine operation at the 200 inch Hale telescope on Palomar mountain. SWIFT is extremely well suited to detailed kinematic and dynamical studies of extended objects. It combines excellent throughput with a substantial (integral) field of view. Thick, fullydepleted CCD detectors provide unsurpassed quantum efficiency at wavelengths up to $1000 \mathrm{~nm}$. With a 2:1 aspect ratio field that permits nodding-on-IFU, it can provide efficient high-quality sky subtraction. In conjunction with the Palomar LGS assisted AO system, it can provide excellent spatial resolution and improved sensitivity at wavelengths shortward of $1 \mu \mathrm{m}$, beyond the traditional range of AO systems. 

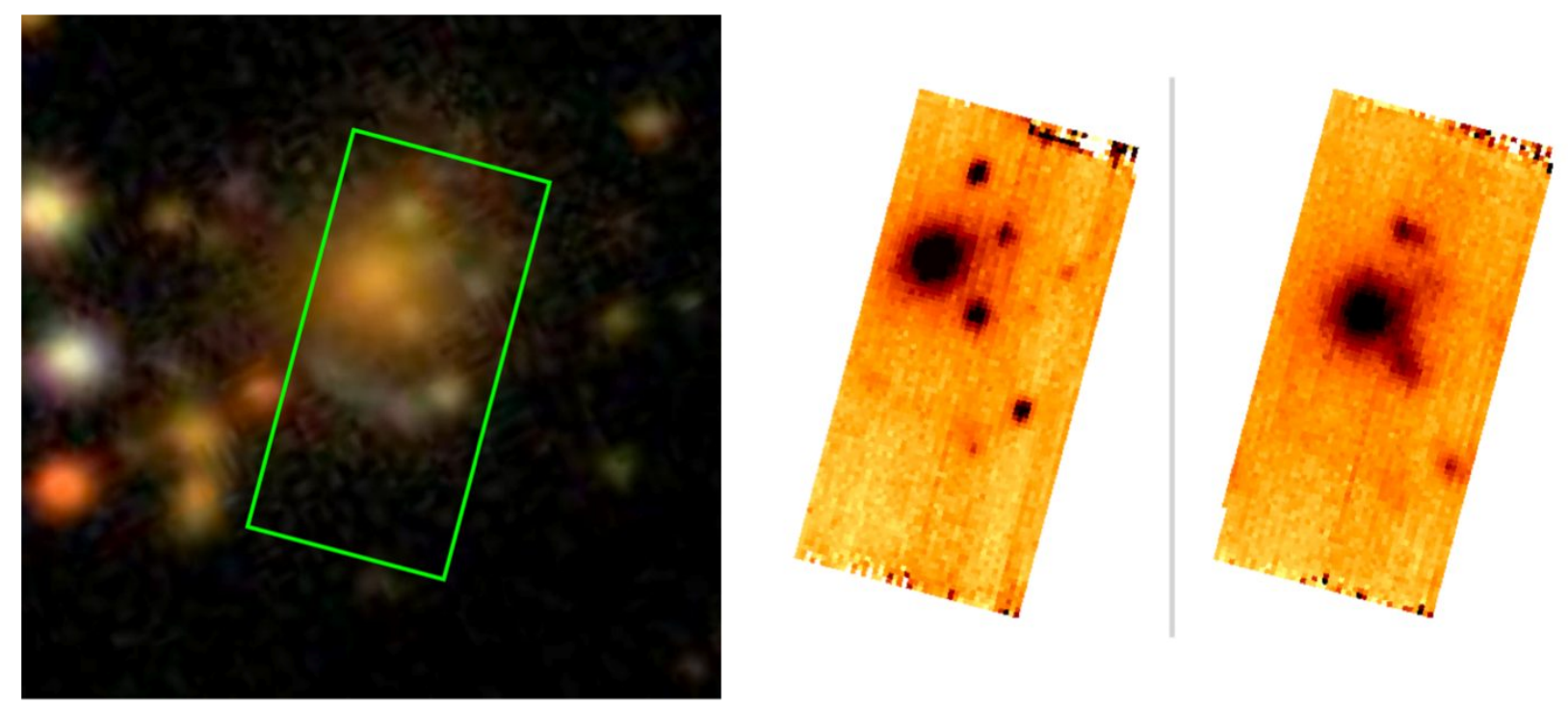

Figure 15: [Left] SDSS image of galaxy cluster with potential lensed arc discovered by a member of the public through the GalaxyZoo project. The green box marks roughly the SWIFT field of view. [Centre] 40 minutes of SWIFT+LGS data on the target (from Aug 2009, seeing 0.8"-1.0") showing the galaxy cluster and potential lens (half way down the SWIFT image. FWHM of the compact objects is around 0.6". [Right] Seeing limited data from May 2009 (seeing 1.2"$\left.1.4^{\prime \prime}\right)$, for comparison. Both images are from a single $600 \mathrm{~s}$ exposure, and were created by collapsing $100 \mathrm{~nm}(\sim 1000$ channels) of the data cube. Data and figures courtesy of A. Verma, M. Tecza \& F. Clarke.

\section{ACKNOWLEDGEMENTS}

The SWIFT team would like to express their sincere gratitude to the Palomar Observatory staff, in particular Steve Kunsman, Mike Doyle, Greg van Idsigna, John Henning and Dan McKenna for their generous help during the installation, commissioning and subsequent operations of the SWIFT spectrograph. We would also like to thank Anna Moore for constructing a foam mock-up of SWIFT several years prior to commissioning, to verify interfaces.

The Oxford SWIFT integral field spectrograph is directly supported by a Marie Curie Excellence Grant from the European Commission (MEXT-CT-2003-002792, Team Leader: N. Thatte). It is also supported by additional funds from the University of Oxford Physics Department and the John Fell OUP Research Fund. Additional funds to host and support SWIFT at the 200-inch Hale Telescope on Palomar are provided by Caltech Optical Observatories. L. Fogarty would like to acknowledge generous support from the Foley-Béjar Scholarship through Balliol College, Oxford and the support of the STFC.

Based on observations obtained at the Hale Telescope, Palomar Observatory, as part of a collaborative agreement between the California Institute of Technology, its divisions Caltech Optical Observatories and the Jet Propulsion Laboratory (operated for NASA), and Cornell University.

\section{REFERENCES}

[1] Dekany, R. et al., "PALM-3000: visible light AO on the 5.1-meter Telescope", Proc. SPIE 6272, 62720G, (2006).

[2] Holland, S. E. et al, "High-voltage-compatable fully depleted CCDs", Proc. SPIE 6276, 62760B, (2006).

[3] Roberts, J. E. et al., "Facilitizing the Palomar AO laser guide star system", Proc. SPIE 7015, 70152S-70152S$10,(2008)$.

[4] Tecza, M. et al., "SWIFT de-magnifying image slicer: diffraction limited image slicing at optical wavelengths", Proc. SPIE 7018, 70182O-70182O-9, (2008).

[5] Thatte, N. et al., "The Oxford SWIFT integral field spectrograph", Proc. SPIE 6269, 62693L, (2006). 
[6] Thatte, N. et al., "SINFONI: a near-infrared AO-assisted integral field spectrometer for the VLT", Proc. SPIE 3353, 704-715, (1998).

[7] Troy, M. et al., "Palomar adaptive optics project: status and performance", Proc SPIE 4007, 31-40, (2000). 